\title{
Highlights of analytical chemical luminescence and cataluminescence
}

\author{
Aldo $\operatorname{Roda}^{1} \cdot \mathrm{Hua} \mathrm{Cui}^{2} \cdot \mathrm{Chao} \mathrm{Lu}^{3}$
}

(C) Springer-Verlag Berlin Heidelberg 2016

"Luminescence" is a generic term that includes all forms of cold light emitted by a molecule in its electronic excited state, which emits light while returning to the electronic ground state. Photoluminescence, particularly fluorescence, is the most widely known and exploited luminescence phenomenon. In photoluminescence, the electronic excited state is formed as a consequence of a physical process (i.e., photoexcitation).

In contrast, "chemical luminescence" refers to those luminescence phenomena whereby light is produced from

Published in the topical collection Highlights of Analytical Chemical Luminescence with guest editors Aldo Roda, Hua Cui, and Chao Lu.

Aldo Roda

aldo.roda@unibo.it

Hua Cui

hcui@ustc.edu.cn

Chao Lu

luchao@mail.buct.edu.cn

1 Department of Chemistry "Giacomo Ciamician”, Alma Mater Studiorum - University of Bologna, Via Selmi 2, 40126 Bologna, Italy

2 CAS Key Laboratory of Soft Matter Chemistry, Collaborative Innovation Center of Chemistry for Energy Materials, Department of Chemistry, University of Science and Technology of China, 230026 Hefei, Anhui, China

3 State Key Laboratory of Chemical Resource Engineering, Beijing University of Chemical Technology, 100029 Beijing, China the dark by a chemical reaction, which yields a product in a singlet excited state.

Chemical luminescence is a fascinating natural phenomenon, which occurs on land and in the ocean, serving a range of functions, from mating to defense. The mechanisms of many bioluminescent species are still a mystery. However, researchers have extensively studied the chemistry of the well-known firefly luciferase bioluminescence, identifying the key intermediates responsible for light emission and coming close to demonstrating the mechanism. It is now known that firefly luciferase bioluminescence involves the enzyme luciferase and the substrate luciferin in an ATP-mediated process.

Nature-inspired chemistry allows researchers to mimic this physiological process by generating new synthetic molecules that decompose with the production of an unstable dioxetanone intermediate in a singlet excited state. This is the key species responsible for light emission. Mimicking nature is a hard job, and most of the chemical luminescence reactions suffer from a low light emission quantum yield, which is much lower than that of the luciferin-luciferase pair.

Chemical luminescence generates much less intense light emission than photoluminescence because the light arises from the molecules involved in the reaction. In contrast, in photoexcitation, the light emission is a function of the excitation light's intensity. Lower light emission intensity may be seen as a disadvantage. However, it can be advantageous when used in bioanalytics. The chemical luminescence light signal may be less intense, but it is also characterized by a very high signal-to- 
noise ratio. This is because the only light background is the thermal noise of the light detector. With the use of new generations of photomultiplier tubes or chargecoupled devices, it is now possible to measure just a few photons, opening up unexpected ways of approaching the development of sensitive and miniaturized analytical devices.

Under the term "chemical luminescence" we include chemiluminescence, bioluminescence, electrogenerated chemiluminescence, and thermochemiluminescence. These phenomena all require a chemical reaction to produce the emitters in the singlet excited state, and this may involve catalysts, enzymes, a redox process, and/or thermodecomposition.

Despite the analytical relevance of chemical luminescence for the development of highly sensitive bioassays and biosensors, the number of publications in the field is much lower than for photoluminescence. Indeed, there are photoluminescence publications covering almost every aspect of analytical chemistry, from separation sciences to imaging.

"Fluorescence" and "photoluminescence" are often used as though they are interchangeable. However, "fluorescence" refers to the excited-state decay mode, which is of course shared by chemical luminescence. In the latter case, the key aspects are chemically generating a singlet excited state instead of a triplet and achieving a reasonable quantum yield of the reaction.

Recent advances in nanotechnology and nanomaterials have facilitated further improvements in chemical luminescence's analytical performance. With this collection, we present the most recent advances in the use of nanoparticles, layered nanomaterials, quantum dots, and new catalysts to improve the quantum efficiency of conventional chemiluminescence systems involving hydrogen peroxide, and related systems.

The contributions to the collection include the use of new chemiluminescence detection concepts to develop new analytical formats for homogeneous immunoassays and for detection of analytes inside cells. Other contributions present new sensitive disposable detectors based on integrated silicon photodiodes, including the use of a smartphone camera to detect the development of portable bioluminescent biosensors. Another aspect addressed by this collection is the availability of new improved bioluminescent proteins, such as the small NanoLuc, which have allowed living whole-cell biosensors to be implemented into mobile biosensing platforms.
Cataluminescence is luminescence that occurs during the course of catalysis. By use of the light signal from the analyte itself, cataluminescence thus offers great analytical potential in terms of real-time response and robustness. It is essentially used for gas sensing, and a wide range of gas-sensing applications can be achieved by the use of different sensor materials. Recent studies have focused mainly on adsorption and the intermediate state of the catalytic process. Nanostructured materials should allow researchers to develop new sensors for gas-phase detection and for detection of analytes in the liquid phase.

We are sure that Analytical and Bioanalytical Chemistry readers will appreciate this collection, which we hope will provide a useful reference for additional studies as well as inspiring new approaches based on nanotechnology and nanomaterials.

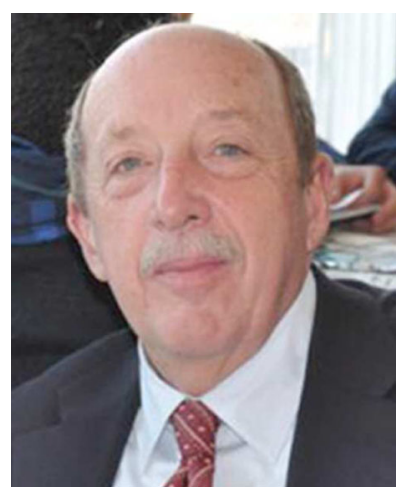

Aldo Roda is Professor of Analytical Chemistry in the Department of Chemistry of Alma Mater Studiorum-University of Bologna, Italy. He is PastPresident of the International Society for Bioluminescence and Chemiluminescence and Vice President of INBB: Biostructures and Biosystems National Institute. $\mathrm{He}$ is an editor of Analytical and Bioanalytical Chemistry and Associate Editor-in-Chief of Luminescence: The Journal of Biological and Chemical Luminescence. He is Secretary of the Academy of Science of Bologna and President of the Chemistry Doctoral School at the University of Bologna.

His main activity focuses on the development of sensitive miniaturized bioassays, immunoassays, biosensors, and imaging based on biochemiluminescence, electrogenerated luminescence, and thermochemiluminescence. Analytical mass spectrometry using matrix-assisted laser desorption ionization and high-performance liquid chromatography-electrospray tandem mass spectrometry is also part of his scientific activity. He has published more than 500 articles, is coauthor of many international patents, and has presented up to 200 invited lectures and oral presentations at international conferences, 


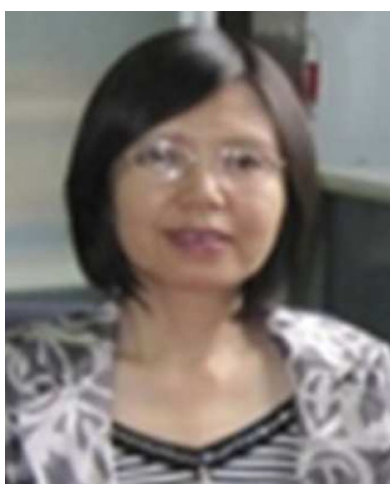

Hua Cui is a full professor of analytical chemistry at the University of Science and Technology of China. She is an editor of Analytical and Bioanalytical Chemistry. Her current research interests are nanochemiluminescence and nanoelectrochemiluminescence, and their applications in interdisciplinary fields, including public health, food safety, and environmental monitoring.

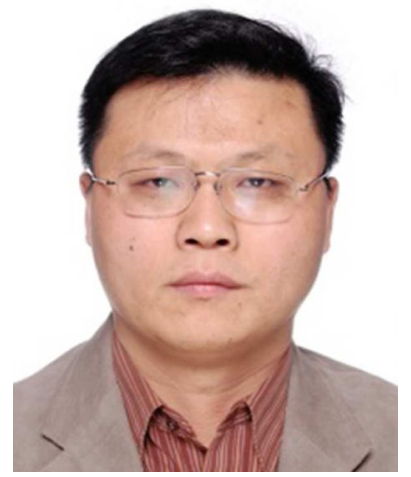

Chao Lu is currently a full professor at the State Key Laboratory of Chemical Resource Engineering, Beijing University of Chemical Technology. In 2011, he was selected to participate in the "New Century Outstanding Talent" scheme of the Ministry of Education. His research interests are focused on the synthesis and characterization of nanostructured materials, nanosensors, and chemiluminescence. He is responsible for national and international research projects, and has published more than 80 articles in peer-reviewed international journals and several patents in the field of analytical and bioanalytical chemistry and nanotechnology. 